\title{
O método diferencial-estrutural: Aplicação para os Estados da Região Sul frente à economia brasileira 1999/2004 e 2004/2008.
}

\author{
Angel dos Santos Fachinelli ${ }^{1}$
}

Umberto Antonio Sesso Filho

Resumo: O objetivo deste artigo foi estudar a dinâmica setorial do emprego nos Estados da Região Sul frente ao Restante do Brasil para os anos de 1999/2004 e 2004/2008. Utilizou-se a matriz da Região Sul e do Restante do Brasil estimadas para o ano de 1999 e 2004 organizadas em 23 setores, juntamente com microdados da Pesquisa Nacional por Amostra de Domicílios (PNAD). Os resultados obtidos através do método shift-share (diferencial-estrutural) identificaram mudanças setoriais na variação teórica do Paraná para os setores Construção Civil e Comércio, em Santa Catarina os setores: Agropecuários, Têxteis e vestuário e Comércio e no Rio Grande do Sul os setores foram Agropecuária e Construção civil. O crescimento do setor terciário em especial no Paraná e em Santa Catarina no segundo período 2004-2008 amenizou a queda de emprego total sofrido pelo Agronegócio e Indústria.

Palavras-chave: Empregos; Região Sul; Método diferencial-estrutural.

JEL: J21, R11, R23

1 Mestre em Economia Regional pela Universidade Estadual de Londrina. Doutoranda em Economia Aplicada pela Escola Superior de Agricultura Luiz de Queiroz (ESALQ/USP). Professora e coordenadora do Curso de Gestão Financeira da Faculdade de Tecnologia da Serra Gaúcha (FTSG). E-mail: angel.fachinelli@ftsg.edu.br 2 Doutor em Economia Aplicada pela Escola Superior de Agricultura Luiz de Queiroz (ESALQ/USP). Professor associado do Departamento de Economia da Universidade Estadual de Londrina (UEL), Pesquisador do CNPq. E-mail: umasesso@uel.br 


\title{
The structural method-differential: Application for States of South Region opposite the Brazilian economy 1999/2004 and 2004/2008.
}

\begin{abstract}
This paper's purpose was to study the dynamics of employment in Brazil's South states against the rest of Brazil's states for the years 1999/2004 and 2004/2008. We used the array for the Southern Region and the Rest of Brazil estimated for the years 1999 and 2004 organized in 23 sectors, with microdata from the National Household Sample Survey (PNAD). The results obtained from the shift-share method (differential-structural) identified sectoral changes in the theoretical variation of Paraná for Trade and Construction sectors, in Santa Catarina the sectors: Agricultural, textiles, clothing and Trade and in Rio Grande do Sul, the main sectors were Agriculture and Construction. The growth of the tertiary sector in particular in Parana and Santa Catarina in the second period 2004-2008 settled down the total employment falling suffered by the Agribusiness and Industry.
\end{abstract}

Keywords: Employment. South Region. Structural-differential method.

JEL: J21, R11, R23

\section{Introdução}

Os estudos temporais que abordam o shift-share (diferencial-estrutural) visam à descrição do crescimento econômico de uma região nos termos de sua estrutura produtiva, composto por um conjunto de identidades que identificam os setores mais dinâmicos e o crescimento destes, ou seja, evidencia quais segmentos vêm perdendo ou adquirindo participação e dinamismo na economia local diante das alterações no cenário nacional.

A fim de avaliar melhor as mudanças setoriais e regionais esse trabalho teve por objetivo identificar as mudanças intra-setoriais e inter-regionais com foco nos Estados da Região Sul (Paraná, Santa Catarina e Rio Grande do Sul) e Restante do Brasil, para tal utilizou-se o método shift-share (diferencial estrutural) nos períodos 1999-2004 e 2004-2008 de modo a compreender melhor as alterações na dinâmica desses Estados.

No período 1999-2008 o Brasil passou por diversos fatos macroeconômicos: crise cambial, instabilidade econômica e políticas de ajustes fiscais, aumento da taxa de juros e proteção aos setores industriais nacionais. A taxa de juros over-selic acumulado segundo relatório do BCB Boletim foi de 23,01\%, 15,14\% e 11,82\% em 1999, 2004 e 2008 respectivamente. A taxa de inflação medida 
pelo IGP-DI (Índice Geral de Preços - disponibilidade interna) obteve percentual em 1999 de 19,98\%, em 2004 de 12,14\% e de 9,10\% em 2008. A taxa de câmbio real/dólar encerrou em 1999 em R $\$ / U \$ 1,78$ e em R $\$ / U \$ 2,65$ em 2004, atingiu sua máxima no ano de 2002 no valor $\mathrm{R} \$ / \mathrm{U} \$ 3,53$ segundo informação do Banco Central do Brasil (BCB, 2011). Estas oscilações impactaram diretamente na comercialização dos produtos no âmbito internacional e indiretamente no mercado interno.

Baltar et al. (2008), na mesma linha de Silva (2008), destacou importantes elementos de continuidade na política macroeconômica brasileira entre o segundo mandato de Fernando Henrique Cardoso e o primeiro governo de Lula, relataram nesta mudança, o efeito da elevação do preço do dólar na dívida pública pelo fato de que, uma parcela expressiva dessa dívida estava indexada à taxa de câmbio, afetando o comércio internacional e nacional.

Alterações no nível tecnológico, ganhos de produtividade, criação de novos parques industriais, são fatores decorrentes de uma melhora no cenário macroeconômico interno, o que se refletiu em especial no período1999-2004. Alterações no cenário macroeconômico internacional e as expectativas futuras em relação à estabilidade política provocaram alterações em especial para os setores ligados a Indústria e a Agropecuária em 2004-2008.

Diante desses fatos, o método shift-share não constitui um modelo comportamental; seu objetivo não é gerar interpretações teóricas, mas sim descrever variações estruturais. Segundo Pereira e Campanile (1999), o crescimento de um setor em determinada região é decomposto em um componente diferencial e outro estrutural.

O presente artigo será distribuído da seguinte maneira: apresenta-se, inicialmente, os estudos regionais utilizando o método de decomposição e, a seguir, o método regional com sua respectiva base de dados, os resultados e discussões em relação às vantagens e desvantagens competitivas especializadas setoriais e regionais nos períodos 1999/2004 e 2004/2008.

\section{Estudos utilizando métodos de decomposição}

Vários aperfeiçoamentos, visando aprimorar deficiências técnicas e conceituais, foram propostos nos últimos 30 anos. Um importante é o de Esteban-Marquillas (1972), que acrescenta o efeito alocação (A), aos efeitos estruturais e diferenciais. $\mathrm{O}$ efeito alocação nos mostraria se a região j está especializada nos setores para os quais dispõe de melhores vantagens competitivas ou não. 
Trabalhos como Brown (1969), que utilizam o método diferencial-estrutural para projeção intertemporal da variável-base. Esteban (1999) procurou elucidar a desigualdade inter-regional na produtividade agregada por trabalhador na União Europeia, utilizou o método shift-share analysis para mostrar que a especialização regional tem um papel muito menor e que as diferenças inter-regionais podem ser explicadas essencialmente pela produtividade uniforme entre os setores.

Lodder (1972) decompôs o crescimento do emprego da economia brasileira, no nível dos Estados e de 30 setores de atividade, incluindo a indústria extrativa, a indústria de transformação, os serviços e a agropecuária. O método foi aplicado para os períodos de 1940-50 e de 1950-60.

Souza (2002) realizou um estudo comparativo das regiões metropolitanas de Curitiba e Belo Horizonte para 1985 e 1997, abordou a análise da dinâmica regional através da metodologia de Análise dos Coeficientes de Importação e Exportação (ACIE) que é uma variante do método Diferencial-Estrutural (shift-share). Entre os principais resultados encontrados destacaram-se as regiões metropolitanas de Belo Horizonte (RMBH) e Curitiba (RMC) tiveram uma inserção positiva na economia nacional. O efeito diferencial que representa a presença da competitividade dos nichos de especialização na RMBH foi muito maior do que o da RMC.

Gonçalves Junior e Galete (2010) analisaram a microrregião de Maringá (MRM) a partir do método Diferencial-Estrutural frente ao estado do Paraná, no período 1994 a 2008. Os autores identificaram que a microrregião apresentou dinamismo, principalmente, nos setores de Confecção de artigos do vestuário e acessórios e Produção de alimentos bebidas.

\section{Método regional shift-share (diferencial-estrutural)}

O método shift-share (diferencial-estrutural) aponta duas razões para o crescimento de uma região: a) a região pode crescer mais que as outras em virtude da sua composição produtiva ser dominada por setores dinâmicos ou b) porque a sua estrutura tem participação crescente no total das regiões, independente da existência de setores. Por estas razões, o crescimento regional é decomposto entre um componente estrutural e um componente diferencial (HADDAD, 1989).

Segundo Souza e Souza (2004) para aplicar o método Diferencial-Estrutural, é necessário a matriz de informações para o ano base e o ano terminal, apresentada no Quadro 1 que ilustra os $m$ setores da economia e as $n$ regiões do 
sistema estadual ou nacional de regiões.

Para dados de emprego, por exemplo, o elemento $E_{21}$ indica o volume de emprego do setor 2 (S2) existente na região 1 (R1); enquanto o elemento $E_{i j}$ se refere ao volume de emprego do setor $i$ na região $j$. No final de cada linha, tem-se o emprego total regional de cada setor $\left(E_{1}, \ldots, E_{m}\right)$; enquanto no final de cada coluna obtém-se o emprego total da região. No encontro da última linha com a última coluna tem-se o emprego total da economia nacional $\left(\sum_{i=1}^{m} E_{i}\right)$.

QUADRO 1 - MATRIZ DE INFORMAÇÕES DAS REGIÕES DO SISTEMA NACIONAL (M SETORES E N REGIÕES)

\begin{tabular}{rrrrrrrrr}
\hline \multirow{2}{*}{ Setores } & \multicolumn{7}{|c}{ Regiões } \\
\cline { 2 - 8 } & $R_{1}$ & $R_{2}$ & $\cdots$ & $R_{j}$ & $\cdots$ & $R_{n}$ & Total Nacio- \\
& $S_{1}$ & $E_{11}$ & $E_{12}$ & $\cdots$ & $E_{1 j}$ & $\cdots$ & $E_{1 n}$ & $E_{1}$ \\
\hline$S_{2}$ & $E_{21}$ & $E_{22}$ & $\cdots$ & $E_{2 j}$ & $\cdots$ & $E_{2 n}$ & $E_{2}$ \\
$\cdots$ & $\cdots$ & $\cdots$ & $\cdots$ & $\cdots$ & $\cdots$ & $\cdots$ & $\cdots$ \\
$S_{i}$ & $E_{i 1}$ & $E_{i 2}$ & $\cdots$ & $E_{i j}$ & $\cdots$ & $E_{i n}$ & $E_{i}$ \\
$\cdots$ & $\cdots$ & $\cdots$ & $\cdots$ & $\cdots$ & $\cdots$ & $\cdots$ & $\cdots$ \\
$S_{m}$ & $E_{m 1}$ & $E_{m 2}$ & $\cdots$ & $E_{m j}$ & $\cdots$ & $E_{m n}$ & $E_{m}$ \\
\hline Total da & $\sum_{i=1}^{m} E_{i 1}$ & $\sum_{i=1}^{m} E_{i 2}$ & $\cdots$ & $\sum_{i=1}^{m} E_{i j}$ & $\cdots$ & $\sum_{i=1}^{m} E_{i n}$ & $\sum_{i=1}^{m} E_{i}$ \\
Região & & & & & & \\
\hline
\end{tabular}

Fonte: Herzog and Olsen, 1977 apud Souza e Souza, 2004.

O objetivo do método é decompor a variação real do emprego de cada setor em cada região, entre o ano base e o ano terminal, para verificar o que se deve ao dinamismo interno regional (efeito diferencial ou regional) e qual a proporção dessa variação que se origina de fatores nacionais (efeito estrutural ou proporcional) ${ }^{1}$. O efeito total $\left(T_{i j}\right)$ será a soma dos efeitos estrutural e diferencial, medindo a diferença entre o crescimento real ou efetivo apresentado pela região e o crescimento teórico ou aquele que a região deveria apresentar caso evoluísse à mesma taxa do país como um todo (SOUZA e SOUZA, 2004).

A estrutura básica do shift-share parte do conceito de variação real do emprego do setor $i$ na região $j\left(\Delta E_{i j}\right)$, entre o ano zero e o ano $t$, é igual ao volume do emprego no ano $t$ do setor $i$ da região $j\left(E_{i j}\right)$, menos o volume do emprego no ano zero desse setor e região $\left(E_{i j}^{0}\right)$ : 


$$
\Delta E_{i j}=E_{i j}^{t}-E_{i j}^{0}
$$

O emprego do ano terminal do setor $i$ na região $j$ pode ser expresso em termos do emprego do ano inicial, multiplicado por sua taxa de crescimento no período $\left(e_{i j}\right)$, como segue:

$$
E_{i j}^{t}=E_{i j}^{0}\left(\frac{E_{i j}^{t}}{E_{i j}^{0}}\right)=E_{i j}^{0} e_{i j}
$$

Substituindo (2) em (1), obtém-se a variação real do emprego do setor $i$ na região $j$ em função do estoque inicial desse emprego e de sua taxa de crescimento $\left(e_{i j}-1\right)$ ou seja:

$$
\Delta E_{i j}=E_{i j}^{0} e_{i j}-E_{i j}^{0}=E_{i j}^{0}\left(e_{i j}-1\right)
$$

Logo a equação (3) pode ser modificada pelas suas respectivas taxas, como: $e=\frac{E^{t}}{E^{0}}$ taxa de crescimento do emprego total (nacional) entre o ano zero e o ano $t ; e_{i}=\frac{E_{i}^{t}}{E^{0}}$ taxa de crescimento do emprego do setor $i$ na região entre o ano zero e o ano $t$. Substituindo essas taxas na equação (3), tem-se:

$$
\begin{aligned}
\Delta E_{i j} & =E_{i j}^{0}\left(e_{i j}-1+e-e+e_{i}-e_{i}\right) \\
& =E_{i j}^{0}\left(e-1+e_{i}-e+e_{i j}-e_{i}\right)
\end{aligned}
$$

Decompondo o segundo membro de (4) em parcelas, tem-se:

$$
\Delta E_{i j}=E_{i j}^{0}(e-1)+E_{i j}^{0}\left(e_{i}-e\right)+E_{i j}^{0}\left(e_{i j}-e_{i}\right)
$$

Substituindo a equação (4) na equação (1), obtém-se:

$$
\Delta E_{i j}=E_{i j}^{t}-E_{i j}^{0}=E_{i j}^{0}(e-1)+E_{i j}^{0}\left(e_{i}-e\right)+E_{i j}^{0}\left(e_{i j}-e_{i}\right)
$$

A equação (6) mostra que a variação real do emprego do setor $i$ na região $j$ $\left(E_{i j}^{t}-E_{i j}^{0}\right)$ pode ser decomposta em três fatores: variação teórica $\left[E_{i j}^{0}(e-1)\right.$ ], variação estrutural $\left[E_{i j}^{0}\left(e_{i}-e\right)\right]$ e variação diferencial $\left[E_{i j}^{0}\left(e_{i j}-e_{i}\right)\right]$. As equações de 1 a 6 correspondem a estrutura básica do método shift-share (diferencial-estrutural), elas contém os dados básicos de emprego por setor de atividade para cada unidade territorial de análise e para o conjunto de regiões (SOUZA e SOUZA, 2000).

Ao rearranjar a equação (6), tem-se o efeito líquido total $\left(T_{i j}\right)$ que poderá ser tanto positivo quanto negativo. Obtém-se a variação líquida pela diferença entre a variação real menos a variação teórica. Logo, o efeito total pode ser decomposto em efeito estrutural e em efeito diferencial:

$$
\begin{aligned}
T_{i j} & =\left(E_{i j}^{t}-E_{i j}^{o}\right)-E_{i j}^{0}(e-1) \\
& =E_{i j}^{0}\left(e_{i}-e\right)+E_{i j}^{0}\left(e_{i j}-e_{i}\right)
\end{aligned}
$$


A equação (7) indica a existência ou não de efeitos estruturais $\left(P_{i j}\right)$ e/ou diferenciais $\left(D_{i j}\right)$ na região $j$, setor por setor. Para computar a existência global da incidência desses efeitos $\left(\sum i T_{i j}\right)$, região por região, é necessário somar os efeitos parciais (positivos e negativos) de todos os setores $i$ da região $j$. Os efeitos estruturas e diferenciais precisam ser calculados para todos os setores do conjunto das regiões Obtém-se a variação líquida total $\left(\sum i T_{i j}\right)$ para cada setor e para cada uma das regiões, fornecendo os efeitos estruturais totais $\left(\sum i P_{i j}\right)$ e os efeitos diferenciais totais $\left(\sum i D_{i j}\right)$. Logo:

$$
\sum i T_{i j}=\sum i P_{i j}+\sum i D_{i j}
$$

A relação (8) indica se a região $j$ apresenta efeitos estruturais $\left(P_{i j}\right)$ e diferenciais $\left(D_{i j}\right)$ positivos ou negativos para todos os setores. Nota-se na equação (8) que os efeitos, estrutural e diferencial, encontram-se entrelaçados, pois ambos dependem do nível do emprego do ano-base $\left(E_{i j}^{0}\right)$. Desse modo, o efeito competitivo também considera aspectos estruturais (HERZOG e OLSEN, 1977 apud SOUZA e SOUZA, 2004).

Esteban-Marquillas (1972) propuseram uma reformulação da equação clássica (6), ao introduzir o emprego esperado $E_{i j}^{0^{*}}$ no lugar do emprego efetivo do período inicial $E_{i j}^{0}$. O emprego esperado para o setor $i$ da região $j\left(E_{i j}^{0^{*}}\right)$ define-se como aquele comporta a mesma proporção da economia nacional para o setor $i$ no ano-base $\left(\frac{E_{i}^{0}}{\sum E_{i}^{0}}\right)$, tal que $\frac{E_{i j}^{\ddagger}}{\sum E_{i j}^{0}}=\frac{E_{i}^{0}}{\sum E_{i}^{0}}$, ou seja:

$$
E_{i j}^{0^{*}}=\sum E_{i j}^{0}\left(\frac{E_{i}^{0}}{\sum E_{i}^{0}}\right)
$$

Ao introduzir $E_{i j}^{0^{*}}$ o efeito diferencial $D_{i j}=E_{i j}^{0}\left(e_{i j}-e_{i}\right)$, no lugar de $E_{i j}^{0}$, procurou-se eliminar da posição competitiva a influência estrutural. Desse modo, a posição competitiva pura é observada em:

$$
D_{i j}^{*}=E_{i j}^{0^{*}}\left(e_{i j}-e_{i}\right)
$$

A influência estrutural do dinamismo diferencial, ou efeito alocação, foi definido como a diferença entre a posição competitiva espúria e a posição competitiva pura $\left(D_{i j}-D_{i j}^{*}=A_{i j}\right)$, obtêm então:

$$
A_{i j}=\left(E_{i j}^{0}-E_{i j}^{0^{*}}\right)\left(e_{i j}-e_{i}\right)
$$

Nessa equação, tem-se o efeito alocação $\left(A_{i j}\right)$, que pode assumir valores positivos, negativos ou nulos, dependendo do sinal. No quadro 4 verifica-se as cinco possibilidades para o sinal de $A_{i j}$, sendo que em três casos o efeito alocação poderá ser nulo. 
QUADRO 2 - SINAIS DOS EFEITOS ALOCAÇÃO DA REGIÃO EM RELAÇÃO À ECONOMIA NACIONAL.

\begin{tabular}{|c|c|c|c|c|}
\hline $\begin{array}{l}\text { Possibilidades para o efeito } \\
\text { alocação }\end{array}$ & Sigla & $\begin{array}{r}\text { Efeito } \\
\text { Alocação } \\
\left(A_{i j}\right)\end{array}$ & $\begin{array}{r}\text { Efeito } \\
\text { Especialização } \\
\left(E_{i j}^{0}-E_{i j}^{0^{*}}\right)\end{array}$ & $\begin{array}{r}\text { Vantagem } \\
\text { Competitiva } \\
\left(e_{i j}-e_{i}\right)\end{array}$ \\
\hline $\begin{array}{l}\text { 1. Desvantagem competitiva } \\
\text { especializada }\end{array}$ & DCE & $(-)$ & $(+)$ & $(-)$ \\
\hline $\begin{array}{l}\text { 2. Desvantagem competiti- } \\
\text { va não especializada }\end{array}$ & $\mathrm{DC} / \mathrm{NE}$ & $(+)$ & $(-)$ & $(-)$ \\
\hline $\begin{array}{l}\text { 3. Vantagem competitiva } \\
\text { não especializada }\end{array}$ & $\mathrm{VC} / \mathrm{NE}$ & $(-)$ & $(-)$ & $(+)$ \\
\hline $\begin{array}{l}\text { 4. Vantagem competitiva } \\
\text { especializada }\end{array}$ & VCE & $(+)$ & $(+)$ & $(+)$ \\
\hline $\begin{array}{l}\text { 5. Neutralidade competitiva } \\
\text { espec./não especializada }\end{array}$ & $\begin{array}{r}\mathrm{NC} / \mathrm{E} \text { ou } \\
\mathrm{NC} / \mathrm{NE}\end{array}$ & o & $(+/-)$ & o \\
\hline
\end{tabular}

Fonte: Herzog and Olsen, 1977 apud Souza e Souza, 2004.

A equação modificada do método Diferencial-Estrutural de Esteban-Marquillas (1972) para um dado setor e região corresponde à:

$$
\begin{aligned}
& \left(E_{i j}^{t}-E_{i j}^{0}\right)= \\
& \quad E_{i j}^{0}(e-1)+E_{i j}^{0}\left(e_{i}-e\right)+E_{i j}^{0^{*}}\left(e_{i j}-e_{i}\right)+\left(E_{i j}^{0}-E_{i j}^{0^{*}}\right)\left(e_{i j}-e_{i}\right)
\end{aligned}
$$

A equação (12) afirma que a variação real $\left(E_{i j}^{t}-E_{i j}^{0}\right)$ é igual à soma das variações teórica $E_{i j}^{0}(e-1)$, estrutural $E_{i j}^{0}\left(e_{i}-e\right)$, competitiva pura $E_{i j}^{0^{*}}\left(e_{i j}-e_{i}\right)$ e alocativa $\left(E_{i j}^{0}-E_{i j}^{0^{0}}\right)\left(e_{i j}-e_{i}\right)$. Os sinais relativos às vantagens ou desvantagens competitivas captadas, bem como os efeitos de alocação e especialização, resultam das principais análises do trabalho empírico.

\subsection{Base de dados}

A base de dados foi obtida dos microdados da Pesquisa Nacional de Amostra por Domicílios (PNAD). A análise dos dados da PNAD de 1999, 2004 e 2008 foi realizada a partir de uma amostra selecionada, que se constitui de indivíduos ocupados no setor urbano e que sejam considerados economicamente ativos.

Para a realização deste trabalho, foram excluídos da amostra os indivíduos com idade inferior a 16 anos ou superior a 65 anos e os indígenas. A classificação da força de trabalho, segundo as posições na ocupação consistiu em: o empregador, autônomo e empregado. 
Na condição da ocupação, consideraram-se como trabalhador formal, os empregados e os trabalhadores domésticos com carteira assinada, militares e funcionários públicos estatutários, empregadores e, como trabalhador informal, os empregados e trabalhadores domésticos sem carteira assinada e trabalhadores autônomos. O software Stata 10 foi utilizado para a retirada das amostras e separação setorial dos dados.

\section{Resultados e discussão}

O estudo do Shift-Share contemplou os Estados do Paraná, Santa Catarina e Rio Grande do Sul frente à economia nacional. A tabela 1 representa a dinâmica estrutural setorial no Estado do Paraná em relação à variação teórica do emprego regional que relata quanto o setor i cresceria na região j se ele se expandisse com a taxa do nível nacional (e).

O estudo verificou que para os anos de 99-04 e 04-08 os setores que mais se destacaram (1) Agropecuária (6,59\%) e (1,96\%), (17) Construção Civil (3,55\%) e $(1,12 \%),(18)$ Comércio $(6,56 \%)$ e $(1,94 \%)$ e o setor (23) Outros serviços $(8,94 \%)$ e $(4,46 \%)$.

O resultado evidenciou os setores mais absorvedores de mão de obra, com elevada rotatividade de funcionários, exigências de qualificação profissional mais baixo e salários médios inferiores ao de categorias com maior exigência de qualificação profissional.

TABELA 1 - VARIAÇÃO $(\Delta)$ TEÓRICA E ALOCAÇÃO $\left(A_{i j}\right)$, POR SETOR ECONÔMICO, PARANÁ.

\begin{tabular}{l|rr|cr}
\hline \multirow{2}{*}{ Setores } & \multirow{2}{*}{$\Delta$ Teórica } & \multicolumn{2}{|c}{ Efeito Alocação } \\
\cline { 4 - 5 } & & & $A_{i j}=\left(E_{i j}^{0}-E_{i j}^{0^{*}}\right)\left(e_{i j}-e_{i}\right)$ \\
\cline { 2 - 5 } & $99-04$ & $04-08$ & $99-04$ & 04-08 \\
\hline 1 Agropecuária & 6,59 & 1,96 & $-3,94$ & $-1,86$ \\
2 Extrativa Mineral & 0,05 & 0,02 & 0,01 & $-0,03$ \\
3 Min. não Metálicos & 0,46 & 0,16 & $-0,01$ & $-0,02$ \\
4 Metal/ Mecânica & 0,58 & 0,25 & $-0,06$ & 0,01 \\
5 Elétrico e Eletrônico & 0,41 & 0,28 & $-0,54$ & $-0,76$ \\
6 Material de Transportes & 0,77 & 0,09 & 0,08 & $-0,02$ \\
7 Madeira e Mobiliário & 1,26 & 0,26 & $-1,10$ & $-0,02$ \\
8 Celulose, Papel e Gráfica & 0,44 & 0,10 & 0,00 & 0,03 \\
9 Indústria da Borracha & 0,01 & 0,14 & $-0,05$ & $-0,13$ \\
\hline Fo:
\end{tabular}

Fonte: Resultados da pesquisa. 
TABELA 1 - VARIAÇÃO $(\Delta)$ TEÓRICA E ALOCAÇÃO $\left(A_{i j}\right)$, POR SETOR ECONÔMICO, PARANÁ. (CONTINUAÇÃO).

\begin{tabular}{|c|c|c|c|c|}
\hline \multirow{3}{*}{ Setores } & \multirow{2}{*}{\multicolumn{2}{|c|}{$\Delta$ Teórica }} & \multicolumn{2}{|c|}{ Efeito Alocação } \\
\hline & & & \multicolumn{2}{|c|}{$A_{i j}=\left(E_{i j}^{0}-E_{i j}^{0^{*}}\right)\left(e_{i j}-e_{i}\right)$} \\
\hline & 99-04 & $04-08$ & 99-04 & $04-08$ \\
\hline 10 Química e Farmácia & 0,31 & 0,17 & 0,03 & $-0,01$ \\
\hline 11 Artigos Plásticos & 0,15 & 0,06 & 0,00 & 0,00 \\
\hline 12 Têxtil e Vestuário & 1,29 & 0,50 & $-0,15$ & 0,04 \\
\hline 13 Fáb. de Calçados & 0,04 & 0,04 & 0,21 & $-0,26$ \\
\hline 14 Ind. Alimentar & 1,11 & 0,47 & $-0,91$ & $-0,07$ \\
\hline 15 Ind. Diversas & 0,17 & 0,27 & $-0,35$ & $-0,41$ \\
\hline 16 SIUP & 0,20 & 0,10 & 0,00 & $\mathrm{O}, \mathrm{OO}$ \\
\hline 17 Construção Civil & 3,55 & 1,12 & 0,10 & 0,23 \\
\hline 18 Comércio & 6,56 & 1,94 & 22,32 & $-62,44$ \\
\hline 19 Transporte & 1,84 & 1,00 & $-0,89$ & $-0,18$ \\
\hline 20 Comunicações & 0,36 & 0,15 & 0,00 & $-0,03$ \\
\hline 21 Inst. Financeiras & 0,50 & 0,28 & $-0,34$ & $-0,08$ \\
\hline 22 Adm. Pública & 2,02 & 1,53 & 2,17 & 0,55 \\
\hline 23 Outros Serviços & 8,94 & 4,46 & $-15,66$ & 3,11 \\
\hline
\end{tabular}

Fonte: Resultados da pesquisa.

A variação diferencial sendo positiva indicou que o setor i cresceu mais na região j do que em nível nacional. Isto estaria indicando vantagens locais para essa atividade na região j. No caso do Paraná, os setores com vantagens locais foram (1) Agropecuária, (5) Elétrico-eletrônico, (14) Indústria alimentar para o período de 99-04.

No período de 04-08 o setor que apresentou vantagem local, foi o setor (18) Comércio. Esses resultados representam a instabilidade econômica da região, de modo que alguns setores perderam participação na economia regional e nacional nesse período, esse efeito diferencial no Estado do Paraná, destacou o setor (18) Comércio em 04-08, possivelmente, por estímulos fiscais, custos nos transportes, diferenças nos preços, fator de produção mais abundante, fez com que esse setor crescesse mais na Região do que no nível nacional.

O quadro 3 ilustra os resultados para os efeitos alocação, especialização e vantagem competitiva para os períodos 1999-2004 e 2004-2008. 
QUADRO 3 - EFEITOS SETORIAIS DO PARANÁ - RESTANTE DO BRASIL

\begin{tabular}{|c|c|c|c|c|c|c|c|c|}
\hline \multirow[t]{2}{*}{ Setores } & \multicolumn{2}{|c|}{$\begin{array}{c}\text { Efeito } \\
\text { Alocação }\end{array}$} & \multicolumn{2}{|c|}{\begin{tabular}{|c|} 
Efeito \\
Especialização \\
\end{tabular}} & \multicolumn{2}{|c|}{$\begin{array}{c}\text { Vantagem } \\
\text { Competitiva }\end{array}$} & \multicolumn{2}{|c|}{ Resultados } \\
\hline & 99-04 & 04-08 & 99-04 & 04-08 & 99-04 & 04-08 & 99-04 & 04-08 \\
\hline 2 & $(+)$ & $(-)$ & $(-)$ & $(-)$ & $(-)$ & $(+)$ & $\mathrm{DC} / \mathrm{NE}$ & VCE \\
\hline 8 & $(+)$ & $(+)$ & $(+)$ & $(+)$ & $(+)$ & $(+)$ & VCE & $\mathrm{VCE}$ \\
\hline 6 & $(+)$ & $(-)$ & $(-)$ & $(-)$ & $(-)$ & $(+)$ & $\mathrm{DC} / \mathrm{NE}$ & $\mathrm{VC} / \mathrm{NE}$ \\
\hline 10 & $(+)$ & $(-)$ & $(-)$ & $(-)$ & $(-)$ & $(+)$ & $\mathrm{DC} / \mathrm{NE}$ & $\mathrm{VC} / \mathrm{NE}$ \\
\hline 13 & $(+)$ & $(-)$ & $(-)$ & $(-)$ & $(-)$ & $(+)$ & $\mathrm{DC} / \mathrm{NE}$ & $\mathrm{VC} / \mathrm{NE}$ \\
\hline 18 & $(+)$ & $(-)$ & $(-)$ & $(-)$ & $(-)$ & $(+)$ & $\mathrm{DC} / \mathrm{NE}$ & $\mathrm{VC} / \mathrm{NE}$ \\
\hline 11 & $(+)$ & $\mathrm{O}$ & $(-)$ & $(-)$ & $(-)$ & $\mathrm{O}$ & $\mathrm{DC} / \mathrm{NE}$ & $\mathrm{NC} / \mathrm{NE}$ \\
\hline 16 & $\mathrm{O}$ & $\mathrm{O}$ & $(+)$ & $(-)$ & $\mathrm{O}$ & $\mathrm{O}$ & $\mathrm{NCE}$ & $\mathrm{NC} / \mathrm{NE}$ \\
\hline 1 & $(-)$ & $(-)$ & $(-)$ & $(+)$ & $(+)$ & $(-)$ & $\mathrm{VC} / \mathrm{NE}$ & DCE \\
\hline 3 & $(-)$ & $(-)$ & $(-)$ & $(+)$ & $(+)$ & $(-)$ & $\mathrm{VC} / \mathrm{NE}$ & DCE \\
\hline 5 & $(-)$ & $(-)$ & $(-)$ & $(+)$ & $(+)$ & $(-)$ & $\mathrm{VC} / \mathrm{NE}$ & DCE \\
\hline 7 & $(-)$ & $(-)$ & $(+)$ & $(+)$ & $(-)$ & $(-)$ & VCE & DCE \\
\hline 9 & $(-)$ & $(-)$ & $(-)$ & $(+)$ & $(+)$ & $(-)$ & $\mathrm{VC} / \mathrm{NE}$ & DCE \\
\hline 14 & $(-)$ & $(-)$ & $(-)$ & $(+)$ & $(+)$ & $(-)$ & $\mathrm{VC} / \mathrm{NE}$ & DCE \\
\hline 15 & $(-)$ & $(-)$ & $(-)$ & $(+)$ & $(+)$ & $(-)$ & $\mathrm{VC} / \mathrm{NE}$ & DCE \\
\hline 19 & $(-)$ & $(-)$ & $(-)$ & $(+)$ & $(+)$ & $(-)$ & $\mathrm{VC} / \mathrm{NE}$ & DCE \\
\hline 20 & $\mathrm{O}$ & $(-)$ & $(+)$ & $(+)$ & $\mathrm{O}$ & $(-)$ & $\mathrm{NCE}$ & DCE \\
\hline 21 & $(-)$ & $(-)$ & $(-)$ & $(+)$ & $(+)$ & $(-)$ & $\mathrm{VC} / \mathrm{NE}$ & DCE \\
\hline 4 & $(-)$ & $(+)$ & $(-)$ & $(-)$ & $(+)$ & $(-)$ & $\mathrm{VC} / \mathrm{NE}$ & $\mathrm{DC} / \mathrm{NE}$ \\
\hline 12 & $(-)$ & $(+)$ & $(-)$ & $(-)$ & $(+)$ & $(-)$ & $\mathrm{VC} / \mathrm{NE}$ & $\mathrm{DC} / \mathrm{NE}$ \\
\hline 17 & $(+)$ & $(+)$ & $(-)$ & $(-)$ & $(-)$ & $(-)$ & $\mathrm{DC} / \mathrm{NE}$ & $\mathrm{DC} / \mathrm{NE}$ \\
\hline 22 & $(+)$ & $(+)$ & $(-)$ & $(-)$ & $(-)$ & $(-)$ & $\mathrm{DC} / \mathrm{NE}$ & $\mathrm{DC} / \mathrm{NE}$ \\
\hline 23 & $(-)$ & $(+)$ & $(-)$ & $(-)$ & $(+)$ & $(-)$ & $\mathrm{VC} / \mathrm{NE}$ & $\mathrm{DC} / \mathrm{NE}$ \\
\hline
\end{tabular}

Fonte: Resultados da pesquisa.

Verificou-se que o último período de análise 2004-2008 no Estado do Paraná os setores: (1) Agropecuária, (3) Minerais não metálicos, (5) Elétrico e eletrônico, , (9) Indústria da Borracha, (14) Indústria alimentar, (15) Indústrias diversas, (19) Transporte, (21) Instituições Financeiras, passaram de vantagem competitiva não especializada (VC/NE) em 1999-2004, para desvantagem competitiva especializada (DCE) 2004-2008.Isso significa que estes setores melhoraram em termos de especialização dentro de sua estrutura, porém, essa melhora ainda não se refletiu em termos de vantagem competitiva em relação 
à taxa de crescimento nacional para o segundo período. Um dos motivos desse efeito seria a taxa de crescimento em nível nacional no segundo período ter sido superior a taxa de crescimento do setor no Paraná.

A produtividade do trabalhador, participação regional no produto nacional e participação dos macro setores na composição do PIB se refletem no estudo. Nesse sentido, observou-se que os setores: (6) Material de transporte, (10) Química e farmácia, (13) Fab. de calçados e (18) Comércio, passaram de DC/ NE entre 1999-2004 para VC/NE entre 2004-2008, estes melhoraram sua vantagem competitiva. Cabe ressaltar que, setores que até 2004 possuíam vantagem competitiva não especializada ( $\mathrm{VC} / \mathrm{NE})$, podem estar perdendo mercado em termos competitivos para outros Estados, os setores (4) Metal/ mecânica e (12) Têxtil e vestuário passaram de (VC/NE) para (DC/NE).

Assim, pode-se verificar para os resultados apresentados na tabela 2 o comportamento das relações de Santa Catarina com o Restante do Brasil em 1999-2004 e 2004-2008

TABELA 2 - VARIAÇÃO $(\Delta)$ TEÓRICA E ALOCAÇÃO $\left(A_{i j}\right)$, POR SETOR ECONÔMICO, SANTA CATARINA.

\begin{tabular}{l|rr|cr}
\hline \multirow{2}{*}{ Setores } & \multirow{2}{*}{$\Delta$ Teórica } & \multicolumn{2}{|c}{ Efeito Alocação } \\
\cline { 4 - 5 } & & & $A_{i j}=\left(E_{i j}^{0}-E_{i j}^{0^{*}}\right)\left(e_{i j}-e_{i}\right)$ \\
\cline { 2 - 5 } & $99-04$ & $04-08$ & $99-04$ & $04-08$ \\
\hline 1 Agropecuária & 5,84 & 1,76 & $-6,31$ & 1,92 \\
2 Extrativa Mineral & 0,08 & 0,02 & 0,03 & 0,00 \\
3 Min. não Metálicos & 0,62 & 0,27 & 0,11 & $-0,01$ \\
4 Metal/ Mecânica & 0,81 & 0,39 & 0,12 & 0,24 \\
5 Elétrico e Eletrônico & 0,74 & 0,30 & 0,13 & 0,11 \\
6 Material de Transportes & 0,45 & 0,08 & $-0,37$ & 0,02 \\
7 Madeira e Mobiliário & 1,62 & 0,47 & $-1,28$ & $-2,45$ \\
8 Celulose, Papel e Gráfica & 0,62 & 0,12 & $-0,13$ & 0,07 \\
9 Indústria da Borracha & 0,01 & 0,09 & $-0,01$ & $-0,01$ \\
10 Química e Farmácia & 0,24 & 0,22 & $-0,17$ & 0,01 \\
11 Artigos Plásticos & 0,71 & 0,12 & $-1,94$ & 0,16 \\
12 Têxtil e Vestuário & 2,91 & 1,11 & $-2,26$ & $-1,99$ \\
13 Fáb. de Calçados & 0,10 & 0,04 & 0,26 & $-0,09$ \\
14 Ind. Alimentar & 1,84 & 0,42 & $-1,88$ & $-0,01$ \\
\hline
\end{tabular}

Fonte: Resultados da pesquisa. 
TABELA 2 - VARIAÇÃO $(\Delta)$ TEÓRICA E ALOCAÇÃO $\left(A_{i j}\right)$, POR SETOR ECONÔMICO, SANTA CATARINA. (CONTINUAÇÃO).

\begin{tabular}{l|rr|r|r}
\hline \multirow{2}{*}{ Setores } & \multicolumn{2}{|c|}{$\Delta$ Teórica } & \multicolumn{2}{c}{ Efeito Alocação } \\
\cline { 4 - 6 } & $99-04$ & $04-08$ & $99-04$ & $04-08$ \\
\cline { 2 - 5 } & 0,23 & 0,40 & $-0,24$ & $-1,72$ \\
\hline 15 Ind. Diversas & 0,34 & 0,11 & $-0,04$ & 0,01 \\
16 SIUP & 3,34 & 1,06 & 0,75 & 1,35 \\
17 Construção Civil & 4,92 & 2,88 & $-26,42$ & $-6,14$ \\
18 Comércio & 1,59 & 0,70 & 0,80 & $-1,07$ \\
19 Transporte & 0,06 & 0,07 & $-0,18$ & $-0,06$ \\
20 Comunicações & 0,49 & 0,23 & $-0,15$ & 0,00 \\
21 Inst. Financeiras & 1,71 & 1,48 & 2,71 & $-8,05$ \\
22 Adm. Pública & 7,59 & 3,51 & 17,69 & 2,71 \\
23 Outros Serviços & & &
\end{tabular}

Fonte: Resultados da pesquisa.

Verificou-se que entre os anos de 1999-2004 a variação teórica positiva mais representativa foi para os setores: (1) Agropecuária (5,84\%), (12) Têxtil e vestuário (2,91\%), (17) Construção civil (3,34\%) e (18) Comércio (4,92\%).

No período que compreende 2004-2008 os setores com maior variação teórica foram: (1) Agropecuária (1,76\%), (12) Têxtil e vestuário (1,11\%) e (18) Comércio $(2,88 \%)$, de modo que estes setores foram os que no emprego cresceram a mesma taxa do conjunto de setores a nível nacional.

Atividades relacionadas ao macro setor indústria tendem a ter um efeito alocação e variação teórica mais estável, visto a dinâmica setorial ser oriunda, em especial, de evoluções e /ou inovações tecnológicas com maior ênfase do que em relação a mão de obra não especializada.

O quadro 4 apresenta os efeitos setoriais de Santa Catarina - Restante do Brasil em 1999-2004 e 2004-2008. Ao se comparar os dois períodos 1999-2004 e 2004-2008 os setores que apresentaram desvantagens locais em termos globais, ou seja, obtiveram taxa de crescimento menor no segundo período em relação ao primeiro foi: (1) Agropecuária, (7) Madeira e Mobiliário, (12) Têxtil e vestuário. Os setores que apresentaram vantagens locais no segundo período em relação ao primeiro foram: (19) Transporte e (22) Administração pública. 
QUADRO 4- EFEITOS SETORIAIS DE SANTA CATARINA - RESTANTE DO BRASIL

\begin{tabular}{r|cc|cccccc}
\hline \multirow{2}{*}{ Setores } & \multicolumn{2}{|c|}{$\begin{array}{c}\text { Efeito } \\
\text { Alocação }\end{array}$} & \multicolumn{2}{c|}{$\begin{array}{c}\text { Efeito } \\
\text { Especialização }\end{array}$} & \multicolumn{2}{c|}{$\begin{array}{c}\text { Vantagem } \\
\text { Competitiva }\end{array}$} & \multicolumn{2}{c}{ Resultados } \\
\cline { 2 - 8 } & $99-04$ & 04-08 & $99-04$ & 04-08 & $99-04$ & 04-08 & 99-04 & 04-08 \\
\hline 4 & $(+)$ & $(+)$ & $(+)$ & $(+)$ & $(+)$ & $(+)$ & VCE & VCE \\
5 & $(+)$ & $(+)$ & $(+)$ & $(+)$ & $(+)$ & $(+)$ & VCE & VCE \\
8 & $(-)$ & $(+)$ & $(+)$ & $(+)$ & $(-)$ & $(+)$ & VCE & VCE \\
11 & $(-)$ & $(+)$ & $(+)$ & $(+)$ & $(-)$ & $(+)$ & VCE & VCE \\
9 & $(-)$ & $(-)$ & $(-)$ & $(-)$ & $(+)$ & $(+)$ & VC/NE & VC/NE \\
13 & $(+)$ & $(-)$ & $(-)$ & $(-)$ & $(-)$ & $(+)$ & DC/NE & VC/NE \\
14 & $(-)$ & $(-)$ & $(+)$ & $(-)$ & $(-)$ & $(+)$ & VCE & VC/NE \\
18 & $(-)$ & $(-)$ & $(-)$ & $(-)$ & $(+)$ & $(+)$ & VC/NE & VC/NE \\
19 & $(+)$ & $(-)$ & $(-)$ & $(-)$ & $(-)$ & $(+)$ & DC/NE & VC/NE \\
20 & $(-)$ & $(-)$ & $(-)$ & $(-)$ & $(+)$ & $(+)$ & VC/NE & VC/NE \\
22 & $(+)$ & $(-)$ & $(-)$ & $(-)$ & $(-)$ & $(+)$ & DC/NE & VC/NE \\
3 & $(+)$ & $(-)$ & $(+)$ & $(+)$ & $(+)$ & $(-)$ & VCE & DCE \\
7 & $(-)$ & $(-)$ & $(+)$ & $(+)$ & $(-)$ & $(-)$ & VCE & DCE \\
12 & $(-)$ & $(-)$ & $(+)$ & $(+)$ & $(-)$ & $(-)$ & VCE & DCE \\
15 & $(-)$ & $(-)$ & $(-)$ & $(+)$ & $(+)$ & $(-)$ & VC/NE & DCE \\
1 & $(-)$ & $(+)$ & $(-)$ & $(-)$ & $(+)$ & $(-)$ & VC/NE & DC/NE \\
2 & $(+)$ & $(+)$ & $(-)$ & $(-)$ & $(-)$ & $(-)$ & DC/NE & DC/NE \\
6 & $(-)$ & $(+)$ & $(-)$ & $(-)$ & $(+)$ & $(-)$ & VC/NE & DC/NE \\
10 & $(-)$ & $(+)$ & $(-)$ & $(-)$ & $(+)$ & $(-)$ & VC/NE & DC/NE \\
16 & $(-)$ & $(+)$ & $(+)$ & $(-)$ & $(-)$ & $(-)$ & VCE & DC/NE \\
17 & $(+)$ & $(+)$ & $(-)$ & $(-)$ & $(-)$ & $(-)$ & DC/NE & DC/NE \\
21 & $(-)$ & $(+)$ & $(-)$ & $(-)$ & $(+)$ & $(-)$ & VC/NE & DC/NE \\
23 & $(+)$ & $(+)$ & $(-)$ & $(-)$ & $(-)$ & $(-)$ & DC/NE & DC/NE \\
\hline
\end{tabular}

Fonte: Resultados da pesquisa.

Destacou-se que em termos de vantagem competitiva o Estado de Santa Catarina perdeu competitividade em setores (1) Agropecuária, (6) Material de Transporte, (10) Química e farmácia e (21) Instituições financeiras, o que é visualizado ao comparar os dois períodos, isso pode ser reflexo da estagnação setorial em relação ao avanço nacional. 
Os setores (3) Minerais não metálicos, (7) Madeira e mobiliário, (12) Textil e Vestuário passaram da situação de Vantagem competitiva especializada para desvantagem competitiva especializada. Pochmann (2006) destacou que, a partir do ano 2000, ocorreu maior ritmo de crescimento do emprego no setor industrial e menor expansão das ocupações tidas como precárias.

A perda de competitividade da agroindústria advém em parte do custo de produção no qual a matéria-prima, problemas portuários, logística podem ter comprometido em especial esses segmentos e são fatores que geram alterações setoriais e locais. A economia regional é sensível também a todo dinamismo da economia nacional, como as mudanças dos fluxos externos de salário. Nesse sentido, o crescimento regional torna-se impulsionado tanto por fatores internos como por fatores externos à região.

Os efeitos setoriais são reflexos também das alterações nas políticas macroeconômicas, mudanças regionais em termos de logística, alterações governamentais e possíveis parcerias fechadas pelos setores dentro da dinâmica regional, ou nas relações interestaduais. Notou-se que para o Estado de Santa Catarina, os setores como (4) Metal/Mecânica, (5) Elétrico e eletrônico, (8) Celulose, papel e Gráfica, (11) Artigos Plásticos não obtiveram alterações em suas estruturas quanto ao grau de especialização e competição. Percebe-se que os setores (4) e (5) são setores diretamente interligados, bem como, os setores (8) e (9). Assim, alterações em um desses setores, causa impacto direto no outro setor.

Esses fatos refletiram nos dados divulgados pela Secretaria de Comércio Exterior (2012), na qual divulga os dados inerentes às importações e exportações brasileiras quanto aos tipos de produtos. Assim, pode-se constatar que para o ano de 1999 os produtos básicos e produtos dos setores da Agropecuária e Pecuária tiveram as maiores quedas nas pautas de exportação, $-8,80$ \% e $-12,32 \%$. Enquanto que, no ano de 2004 as exportações de produtos básicos totalizaram 34,66\% da pauta de exportações e a Agropecuária 31,22\%.

O Estado de Santa Catarina também aumentou sua participação regional no produto interno bruto nacional em termos percentuais ao longo dos anos. Em 1999 o Estado representou 3,5\% no total do PIB enquanto que em 2008 sua participação foi de 4,10\% (IBGE, 2012).

Os Estados da Região Sul são diferentes em suas estruturas. Assim, a tabela 3 reporta os resultados da variação teoria e alocação por setor econômico entre 1999-2004 e 2004-2008 para o Estado do Rio Grande do Sul. 
TABELA 3 - VARIAÇÃO $(\Delta)$ TEÓRICA E ALOCAÇÃO ( $A_{i j}$ AIJ), POR SETOR ECONÔMICO, ENTRE 1999-2004 E 2004-2008 ESTADO DO RIO GRANDE DO SUL.

\begin{tabular}{|c|c|c|c|c|}
\hline \multirow{3}{*}{ Setores } & \multirow{2}{*}{\multicolumn{2}{|c|}{$\Delta$ Teórica }} & \multicolumn{2}{|c|}{ Efeito Alocação } \\
\hline & & & \multicolumn{2}{|c|}{$A_{i j}=\left(E_{i j}^{0}-E_{i j}^{0^{*}}\right)\left(e_{i j}-e_{i}\right)$} \\
\hline & $99-04$ & $04-08$ & 99-04 & 04-08 \\
\hline 1 Agropecuária & 5,57 & 1,76 & $-23,49$ & 1,92 \\
\hline 2 Extrativa Mineral & 0,13 & 0,02 & 0,00 & 0,00 \\
\hline 3 Min. não Metálicos & 0,28 & 0,27 & $-0,03$ & $-0,01$ \\
\hline 4 Metal/ Mecânica & 0,73 & 0,39 & $\mathrm{O}, \mathrm{OO}$ & 0,24 \\
\hline 5 Elétrico e Eletrônico & 0,69 & 0,30 & 0,12 & 0,11 \\
\hline 6 Material de Transportes & 0,59 & 0,08 & $-0,24$ & 0,02 \\
\hline 7 Madeira e Mobiliário & 0,91 & 0,47 & $-0,32$ & $-2,45$ \\
\hline 8 Celulose, Papel e Gráfica & 0,28 & 0,12 & $-0,10$ & 0,07 \\
\hline 9 Indústria da Borracha & 0,07 & 0,09 & $-0,02$ & $-0,01$ \\
\hline 10 Química e Farmácia & 0,32 & 0,22 & 0,08 & $\mathrm{O}, \mathrm{O} 1$ \\
\hline 11 Artigos Plásticos & 0,16 & 0,12 & 0,00 & 0,16 \\
\hline 12 Têxtil e Vestuário & 1,02 & 1,11 & 0,98 & $-1,99$ \\
\hline 13 Fáb. de Calçados & 2,03 & 0,04 & 3,30 & $-0,09$ \\
\hline 14 Ind. Alimentar & 1,61 & 0,42 & $-0,24$ & $-0,01$ \\
\hline 15 Ind. Diversas & 0,28 & 0,40 & 0,00 & $-1,72$ \\
\hline 16 SIUP & 0,16 & 0,11 & $-0,10$ & $\mathrm{O}, 01$ \\
\hline 17 Construção Civil & 3,06 & 1,06 & $-0,19$ & 1,35 \\
\hline 18 Comércio & 5,99 & 2,88 & $-0,66$ & $-6,14$ \\
\hline 19 Transporte & 1,55 & 0,70 & $-0,11$ & $-1,07$ \\
\hline 20 Comunicações & 0,23 & 0,07 & $-0,02$ & $-0,06$ \\
\hline 21 Inst. Financeiras & 0,63 & 0,23 & 0,02 & 0,00 \\
\hline 22 Adm. Pública & 2,28 & 1,48 & 1,71 & $-8,05$ \\
\hline 23 Outros Serviços & 8,80 & 3,51 & 20,66 & 2,71 \\
\hline
\end{tabular}

Fonte: Resultados da pesquisa.

A variação teórica do Estado obteve resultado positivo mais expressivo entre 1999-2004 nos setores: (1) Agropecuária (5,57\%), (13) Fabricação de calçados (2,03\%), (17) Construção Civil (3,06\%), (18) Comércio (5,99\%), (22) Administração Pública (2,28\%), (23) Outros serviços (8,80\%).

No período que compreende 2004-2008 a variação teórica com maior desta- 
que nos setores foram: (1) Agropecuária (1,76\%), (13) Fabricação de calçados (0,04\%), (17) Construção Civil (1,06\%), (18) Comércio (2,88\%), (22) Administração pública (1,48\%), (23) Outros serviços. Portanto, verificou-se que no período de 2004-2008 houve uma melhora no desempenho econômico desses setores no Rio Grande do Sul.

No que tange ao efeito diferencial, cabe ressaltar que setores que apresentam sinais positivos, normalmente, indicam a existência de maiores facilidades de acesso ao mercado consumidor, aos fatores de produção, inovação tecnológica, entre outros fatores que favoreceram tal setor em relação ao mesmo setor em outra região.

O Quadro 5 ilustra-se os efeitos setoriais do Rio Grande do Sul - Restante do Brasil em 1999-2004 e 2004-2008.

As possibilidades do efeito alocação para o Estado do rio Grande do sul indicaram que o Estado sofreu alterações como de destaque nos setores: (1) Agropecuária, (6) Material de Transporte, passando de VC/NE para DC/NE o que enuncia uma estagnação desses setores em relação ao processo de melhoria e/ou crescimento operacional e tecnológico do restante do país, nesses casos os setores (1) Agropecuária e (6) Material de transporte melhoraram o efeito alocação, porém com perdas de competitividade, boa parte dessa perda de competitividade advém da falta de infraestrutura, pedágios que encarecem o frete, e a logística do Estado que contempla boa parte de suas estradas com curvas sinuosas, devido ao mapeamento geográfico da região.

Verificou-se também que setores como (13) Fabricação de calçados e (14) Indústria alimentar que passaram de VCE para VC/NE obtiveram perdas nas especializações, porém se tornaram mais competitivos, nesse período houve migrações de empresas calçadistas na região para outros Estados Brasileiros.

Os setores (3) Min. não Metálicos, (12) Têxtil e Vestuário e (15) Ind. Diversas avançaram no quesito especialização. Entre 1990 e 1998, por exemplo, com a abertura econômica do Brasil, o nível de emprego caiu em setores ligados ao macro setor industriais, a modernização agrícola também contribuiu para reduzir o número de empregos, visto que, processos tecnológicos são poupadores de trabalho, principalmente nos setores de maior concorrência, estes fatos obtiveram uma contrapartida do setor terciário que absorveu em parte a mão de obra oriunda desses setores.

No Rio Grande do Sul, não muito diferente dos demais Estados da Região Sul, pode estar perdendo dinamismo apesar de sua especialização nos setores (13)Fabricação de Calçados (14) Indústria Alimentar. No caso de empresas na área de calçados, verificou-se a migração de algumas empresas calçadistas do Rio Grande do Sul para a Região do Nordeste em busca de incentivos fiscais, mão de obra mais barata e menos intervenção sindical, no caso do setor alimentício, percebeu-se que problemas de logística, clima e falta de 
incentivo estadual fez com que o setor de alimentos perde-se especialização. QUADRO 5- EFEITOS SETORIAIS DO RIO GRANDE DO SUL - RESTANTE DO BRASIL EM 1999-2004 E 2004-2008.

\begin{tabular}{r|rrrrrrrrr}
\hline \multirow{2}{*}{ Setores } & \multicolumn{2}{|c|}{$\begin{array}{c}\text { Efeito } \\
\text { Alocação }\end{array}$} & \multicolumn{2}{c|}{$\begin{array}{c}\text { Efeito } \\
\text { Especialização }\end{array}$} & \multicolumn{2}{c|}{$\begin{array}{c}\text { Vantagem } \\
\text { Competitiva }\end{array}$} & \multicolumn{2}{c}{ Resultados } \\
\cline { 2 - 9 } & $99-04$ & 04-08 & $99-04$ & 04-08 & $99-04$ & 04-08 & $99-04$ & 04-08 \\
\hline 4 & $(+)$ & $(+)$ & $(+)$ & $(+)$ & $(+)$ & $(+)$ & VCE & VCE \\
5 & $(+)$ & $(+)$ & $(+)$ & $(+)$ & $(+)$ & $(+)$ & VCE & VCE \\
8 & $(-)$ & $(+)$ & $(-)$ & $(+)$ & $(+)$ & $(+)$ & VC/NE & VCE \\
11 & o & $(+)$ & $(-)$ & $(+)$ & 0 & $(+)$ & NC/NE & VCE \\
9 & $(-)$ & $(-)$ & $(+)$ & $(-)$ & $(-)$ & $(+)$ & VCE & VC/NE \\
13 & $(+)$ & $(-)$ & $(+)$ & $(-)$ & $(+)$ & $(+)$ & VCE & VC/NE \\
14 & $(-)$ & $(-)$ & $(+)$ & $(-)$ & $(-)$ & $(+)$ & VCE & VC/NE \\
18 & $(-)$ & $(-)$ & $(-)$ & $(-)$ & $(+)$ & $(+)$ & VC/NE & VC/NE \\
19 & $(-)$ & $(-)$ & $(-)$ & $(-)$ & $(+)$ & $(+)$ & VC/NE & VC/NE \\
20 & $(-)$ & $(-)$ & $(-)$ & $(-)$ & $(+)$ & $(+)$ & VC/NE & VC/NE \\
22 & $(+)$ & $(-)$ & $(-)$ & $(-)$ & $(-)$ & $(+)$ & DC/NE & VC/NE \\
21 & $(+)$ & 0 & $(-)$ & $(-)$ & $(-)$ & 0 & DC/NE & NC/NE \\
3 & $(-)$ & $(-)$ & $(-)$ & $(+)$ & $(+)$ & $(-)$ & VC/NE & DCE \\
7 & $(-)$ & $(-)$ & $(+)$ & $(+)$ & $(-)$ & $(-)$ & VCE & DCE \\
12 & $(+)$ & $(-)$ & $(-)$ & $(+)$ & $(-)$ & $(-)$ & DC/NE & DCE \\
15 & 0 & $(-)$ & $(-)$ & $(+)$ & 0 & $(-)$ & NC/NE & DCE \\
1 & $(-)$ & $(+)$ & $(-)$ & $(-)$ & $(+)$ & $(-)$ & VC/NE & DC/NE \\
2 & 0 & $(+)$ & $(-)$ & $(-)$ & 0 & $(-)$ & NC/NE & DC/NE \\
6 & $(-)$ & $(+)$ & $(-)$ & $(-)$ & $(+)$ & $(-)$ & VC/NE & DC/NE \\
10 & $(+)$ & $(+)$ & $(-)$ & $(-)$ & $(-)$ & $(-)$ & DC/NE & DC/NE \\
16 & $(-)$ & $(+)$ & $(-)$ & $(-)$ & $(+)$ & $(-)$ & VC/NE & DC/NE \\
17 & $(-)$ & $(+)$ & $(-)$ & $(-)$ & $(+)$ & $(-)$ & VC/NE & DC/NE \\
23 & $(+)$ & $(+)$ & $(-)$ & $(-)$ & $(-)$ & $(-)$ & DC/NE & DC/NE \\
\hline
\end{tabular}

Fonte: Resultados da pesquisa.

Setores consolidados na região como (4) Metal/ Mecânico, (5) Eletro/eletrônico, fortes em especial na região da Serra Gaúcha, mantiveram a vantagem competitiva especializada, puxando os setores relacionados como (5) Elétrico Eletrônico e o setor (11) Artigos plásticos que melhoraram sua especialização para atender a necessidade dos demais setores relacionais.. 
Conforme o trabalho de Souza e Souza (2000) o alto custo dos terrenos e dos aluguéis, o custo da mão de obra também provoca o deslocamento de atividades para outras regiões, como a produção de calçados e de produtos têxteis, que estão se implantando em Estados do Nordeste, outro fato explicitado pelo autor é em relação a alguns municípios próximos a capital como Canoas, Guaíba, Esteio e São Leopoldo, Novo Hamburgo, Estância Velha, Ivoti e Triunfo mostraram desvantagem competitiva e são municípios que possuem uma estrutura industrial consolidada, mas que também perderam dinamismo e vantagem competitiva em determinados setores para regiões distantes da capital.

Os incentivos fiscais, logística, custo da mão de obra mais baixo e retornos do capital investido mais expressivos alteraram a dinâmica regional, alterando o cenário local o que explica em parte algumas das principais mudanças nos dois períodos em todas as regiões.

\section{Considerações Finais}

No processo de integração econômica as peculiaridades regionais têm-se mostrado muito úteis para proporcionar inserções vantajosas, em especial, as formas pelas quais as regiões buscam o desenvolvimento são inúmeras e variadas, tornando sem sentido a procura por "modelos generalizáveis", as peculiaridades tendem a ser valorizadas tornando as regiões com vantagem competitivas e especializadas o ponto chave da evolução regional e setorial.

A instabilidade regional no período ficou evidente em todas as regiões estudadas, de modo que setores que apresentaram vantagens locais no primeiro período não apresentaram, necessariamente no segundo. Os fatos que justificam essas alterações devem ser analisados separadamente, cada setor possui as suas peculiaridades e as políticas regionais afetam diretamente a tomada de decisão por parte do empresário.

Os resultados explicitam as diferenças nas economias regionais, a sensibilidade destas ao dinamismo da economia nacional, como a mudanças dos fluxos externos de salário, emprego e produção. Para finalizar, este trabalho buscou avaliar de uma maneira ampla e genérica, o comportamento setorial nos Estados da Região Sul em comparação com as taxas de crescimento em nível nacional.

Pode-se concluir que a Região Sul perdeu dinamismo em setores ligados ao Agronegócio e a Indústria e ganhou em outros setores relacionados ao Comércio e Serviços, com ressalvas para cada Estado, sendo de suma importância uma análise detalhada em nível de setor regional no que diz respeito a futuros 
trabalhos. Um complemento em relação às ideias e conclusões já expostas, explorando as diferenças regionais, subsidiaria e evidenciaria a necessidade de políticas específicas para os setores e regiões.

\section{Referências Bibliográficas}

BALTAR,P.A.; SANTOS.A.L.; KREIN. J.D.; LEONE.E.; PRONI.M.W.; MORETTO.A.; MAIA.A.G.; SALAS.C. (2010). "Trabalho no governo Lula: uma reflexão sobre a recente experiência brasileira.” Global Labour University Working Papers., n.9.

BANCO CENTRAL DO BRASIL (BCB) (2011). Selic mercado de títulos públicos. URL [on-line]: < http://www.bcb.gov.br/?SELIC>.

BROWN, H. J.(1969). "Shift and share projection of regional economic growth: an empirical test.” JRS, v. 9(1).

ESTEBAN-MARQUILLAS, J. M. A . (1972). "A reinterpretation of shift-share analysis.” Regional and Urban Economics, v.2(3), pp. 49-55.

ESTEBAN, J. (1999). "Regional Convergence in Europe and the Industry-Mix: a Shift-Share Analysis.” Institut d'Anàlisi Econòmica, CSIC. Campus de la UAB Bellaterra. Barcelona.

FACULDADE GETÚLIO VARGAS (FGV). (2011). Índices gerais de preços. URL [on-line]: <//portalibre.fgv.br/> Acesso em: mai. de 2011.

GONÇALVES JUNIOR, C. A.; GALETE, R. A. (2010). "O método estrutural-diferencial: aplicação da adaptação de Herzog e Olsen para a microrregião de Maringá frente à economia paranaense 1994/2008.” Informe Gepec, Toledo, v. 14 (2), pp. 149-165.

HADDAD, P. R. (Org.) (1989). “Economia regional, teorias e métodos de análise. “ In: HADDAD, P. R. Fortaleza Ç BNB;ETENE.

HERZOG, Henry W.; OLSEN, R. J. (1977). "Shift-Share Analysis Revisited: The Allocation Effect and the Stability of Regional Structure.” Journal of Regional Science, v.17 (3), pp.441-454.

IBGE. (2012). Compatibilização entre as atividades do Sistema de Contas Nacionais - SCN e Classificação Nacional das Atividades - CNAE.

. (1999). Pesquisa Nacional por Amostra de Domicílios - PNAD. Rio de Janeiro: IBGE, CD-ROM.

. (2004). Pesquisa Nacional por Amostra de Domicílios - PNAD. Rio de Janeiro: IBGE,CD-ROM.

(2008). Pesquisa Nacional por Amostra de Domicílios - PNAD. Rio de Janeiro: IBGE,CD-ROM

LODDER, C. A. (1972). “Crescimento da ocupação regional e seus componentes.” In: HADDAD, P.R. (ed.). Planejamento Regional: métodos e aplicação ao caso brasileiro. Rio de Janeiro: IPEA/ INPES. 
MINISTÉRIO DO DESENVOLVIMETO INDÚSTRIA E COMÉRCIO EXTERIOR. Secretaria do Comércio Exterior (SECEX). (2012). URL [on-line]: <//mdic.gov. $\mathrm{br} /$ sitio/secex/secex/informativo.php>.

PEREIRA, A. S.; CAMPANILE, N. (1999). “O método estrutural-diferencial modificado: uma aplicação para o estado do Rio de Janeiro entre 1986 e 1995.” Revista Teoria e Evidência Econômica, v. 7 (13), pp. 121-140.

POCHMANN, M. (2006). "Mercado geral de trabalho: o que há de novo no Brasil?” Revista Parcerias Estratégicas, CGEE, n. 22.

SILVA, Walter Franco L. da. (2008). Macroambiente e cenários econômicos. IESDE Brasil S.A.

SOUZA, C. C. A. (2002). “Análise do crescimento das regiões metropolitanas de Belo Horizonte e Curitiba: um estudo comparativo.” In: Anais do X Seminário sobre a Economia Mineira, Cedeplar-MG.

SOUZA, J.; SOUZA, R. B L de. (2004). "Dinâmica estrutural-diferencial da Região Metropolitana de Porto Alegre, 1990/200o.” Revista de Economia. Curitiba.

Recebido em: 4 de novembro de 2013

Aceito em: 5 de maio de 2014 
